Supporting Information

\title{
Distinct and Selective Amine- and Anion-Responsive Behaviors of an Electron-Deficient and Anion-Exchangeable Metal-Organic Framework
}

Meng-Yue Guo, ${ }^{\dagger \uparrow}$ Peng Li, ${ }^{\dagger \uparrow}$ Shuai-Liang Yang, ${ }^{\dagger}$ Ran Bu, ${ }^{\dagger}$ Xian-Qing Piao, ${ }^{\dagger}$ En-Qing Gao *广

†Shanghai Key Laboratory of Green Chemistry and Chemical Processes, School of Chemistry and Molecular Engineering, East China Normal University, Shanghai 200062, China.

*Engineering Research Center for Nanophotonics and Advanced Instrument, School of Physics and Electronic Science, East China Normal University, Shanghai 200062, China.

${ }^{\S}$ College of Chemistry and Materials Science, Huaibei Normal University, Anhui 235000, China 


\section{Physical Measurements}

Powder X-ray diffraction (PXRD) was recorded on a Rigaku D/Max-2500 diffractometer at $40 \mathrm{kV}, 30 \mathrm{~mA}$ for a $\mathrm{Cu}$-target tube and a graphite monochromator in a $2 \theta$ range of $5-30^{\circ}$ at room temperature. FT-IR spectra were recorded in the range $500-4000 \mathrm{~cm}^{-1}$ using $\mathrm{KBr}$ pellets on a Nicolet NEXUS 670 spectrophotometer. Thermal gravimetric analysis (TGA) was performed on a STA $449 \mathrm{~F} 3$ Simultaneous Thermal Analyzer in flowing air at $10^{\circ} \mathrm{C} / \mathrm{min}$. NMR spectra were recorded on a Bruker Advance $400 \mathrm{MHz}$ spectrometer. UV-vis spectra were obtained on a HIMADZU UV-2700 spectrometer. $\mathrm{BaSO}_{4}$ plates were used as references $(100 \%$ reflection), on which the finely ground power of a sample was coated. Electron spin resonance (ESR) spectra were recorded on a Bruker Elexsys 580 spectrometer with a $100 \mathrm{kHz}$ magnetic field in the $\mathrm{X}$ band at room temperature.

\section{Crystal structure determination}

Data collection was performed using a Bruker SMART APEXII Diffraction diffractometer $\left(\mathrm{Cu}-\mathrm{K}_{\alpha}\right.$ radiation, $\lambda=1.54184 \AA$ ) equipped with a graphite monochromator and a CCD area detector. Structure solution was carried out using the direct method, and full-matrix leastsquares refinements were performed on $F^{2}$ with the SHELX-2014 program. ${ }^{1}$ Non-hydrogen atoms were all refined an isotropically. The $\mathrm{H}$ atoms attached to carbons were added geometrically and refined isotropically with the riding model, and those of the coordinated water molecules were located from the difference map and refined with distance restraints. The chloride ions in the pores of the compound were located, some of which were disordered over two positions with refined occupancies. The coordinated water molecules were located but the free and heavily disordered solvent molecules were not. The final refinements were performed after subtracting the intensity contribution from the solvent molecules using the "SQUEEZE" protocol of the PLATON program. ${ }^{2}$ The crystallographic data and the parameters for the final refinement are presented in Table S1. The content of the solvents was determined from elemental analysis and thermogravimetric measurements (Figure S1). 
Table S1. Crystal data and structure refinement for $\mathbf{1}$.

\begin{tabular}{|c|c|}
\hline Compound & 1 \\
\hline Empirical formula & $\mathrm{C}_{88} \mathrm{H}_{86} \mathrm{Cl}_{8} \mathrm{Eu}_{4} \mathrm{~N}_{8} \mathrm{O}_{25}$ \\
\hline Formula mass & 2547.08 \\
\hline Crystal system & Triclinic \\
\hline Space group & $P 1$ \\
\hline$a(\AA)$ & $10.4971(3)$ \\
\hline$b(\AA)$ & $16.3367(5)$ \\
\hline$c(\AA)$ & 19.0994(5) \\
\hline$\alpha\left(^{\circ}\right)$ & 84.8450 \\
\hline$\beta\left(^{\circ}\right)$ & 76.7760 \\
\hline$\gamma\left({ }^{\circ}\right)$ & 86.8570 \\
\hline$V\left(\AA^{3}\right)$ & $3173.59(16)$ \\
\hline$Z$ & 1 \\
\hline$T / \mathrm{K}$ & 296(2) \\
\hline $\mathrm{D}_{\text {calcd }}\left(\mathrm{g} \cdot \mathrm{cm}^{-3}\right)$ & 1.333 \\
\hline$\mu\left(\mathrm{mm}^{-1}\right)$ & 15.97 \\
\hline Reflections collected & 43946 \\
\hline Unique Reflections $\left(\mathrm{R}_{\text {int }}\right)$ & $21855(0.043)$ \\
\hline No. Observations $(\mathrm{I}>2 \sigma(\mathrm{I}))$ & 20718 \\
\hline No. Variables & 1268 \\
\hline $\mathrm{R}_{1}^{[\mathrm{a}]}, \mathrm{wR}_{2}{ }^{[\mathrm{b}]} \quad(\mathrm{I}>2 \sigma(\mathrm{I}))$ & $0.038,0.101$ \\
\hline $\mathrm{GOF}^{\mathrm{c}}$ & 1.02 \\
\hline$\Delta / \rho_{\max }\left(\mathrm{e} / \AA^{3}\right)$ & 0.90 \\
\hline$\Delta / \rho_{\min }\left(\mathrm{e} / \AA^{3}\right)$ & -0.81 \\
\hline
\end{tabular}

${ }^{[\mathrm{a}]} R=|| F_{\mathrm{o}}|-| F_{\mathrm{c}}|/| F_{\mathrm{o}} \mid .{ }^{\left[{ }^{\mathrm{b}}\right]} R w=\left\{w\left(F_{\mathrm{o}}{ }^{2}-F_{\mathrm{c}}{ }^{2}\right)^{2} / w\left(F_{\mathrm{o}}\right)^{2}\right\}^{1 / 2} .{ }^{[\mathrm{c}]} \mathrm{GOF}=\left\{w\left(\left(F_{\mathrm{o}}{ }^{2}-F_{\mathrm{c}}{ }^{2}\right)^{2}\right) /(n-p)\right\}^{1 / 2}$, where $n=$ number of reflections and $p=$ total numbers of parameters refined.

Table S2. Parameters for hydrogen bonds.

\begin{tabular}{llllll}
\hline D-H...A & D-H & H...A & D...A & $<($ DHA $)$ & \\
\hline O13-H13B...O3 & 0.82 & 1.90 & $2.700(8)$ & 165.1 & \\
O19-H19B...O3 & $0.86(3)$ & $1.95(6)$ & $2.771(12)$ & $158(13)$ & intraframework \\
O21-H21B...O3(x-1, y, z) & $0.87(3)$ & $1.99(5)$ & $2.826(11)$ & $161(12)$ & interframework \\
\hline
\end{tabular}




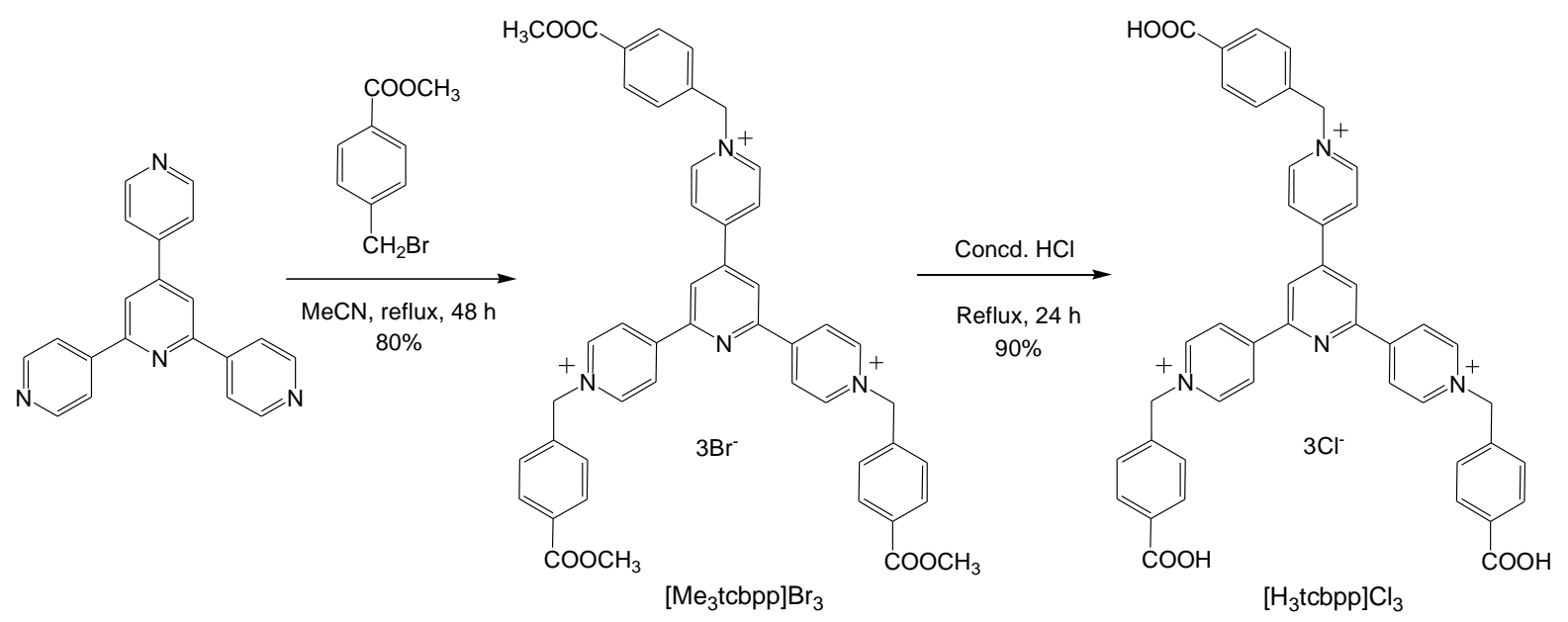

Scheme S1. Synthesis of ligand $\left[\mathrm{H}_{3} \mathrm{tcbpp}\right] \mathrm{Cl}_{3}$.

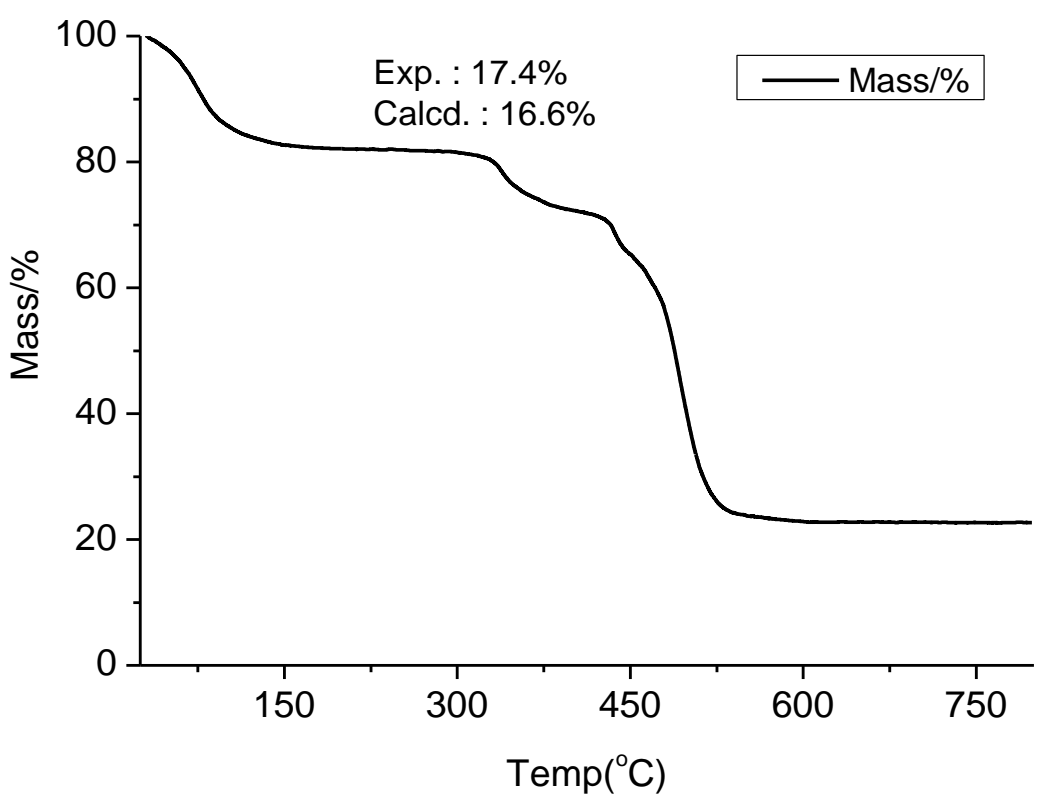

Figure S1. Thermogravimetric plot of 1 from room temperature to $800{ }^{\circ} \mathrm{C}$ under air atmosphere. The weight loss $(\sim 17.4 \%)$ upon heating to $150{ }^{\circ} \mathrm{C}$ corresponds to the release of all solvent molecules (calcd. 16.6\%). 
(a)

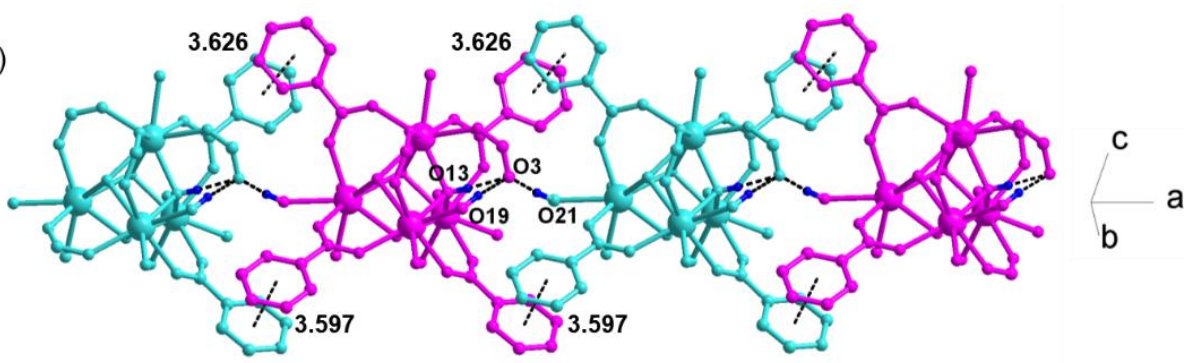

(b)

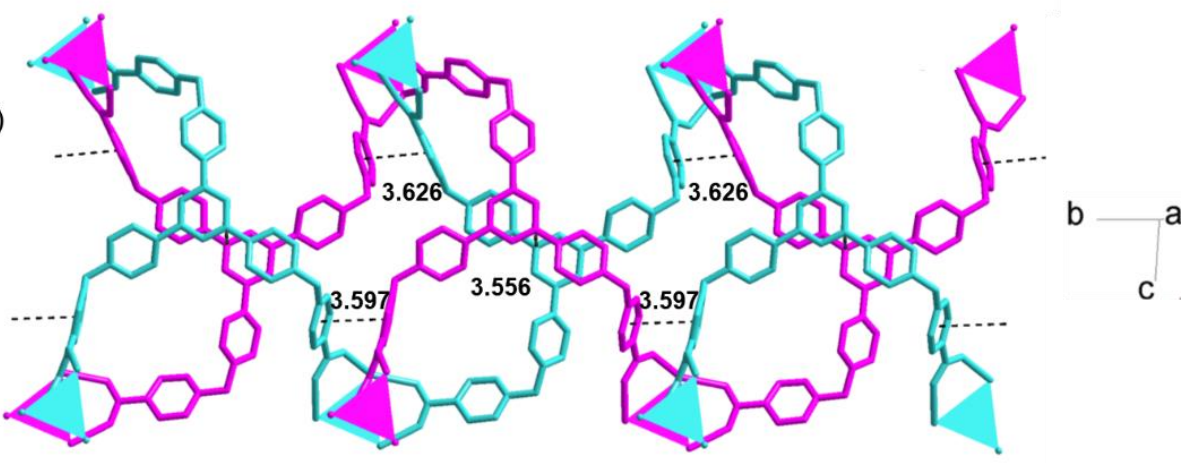

Figure S2. The $\pi-\pi$ stacking and hydrogen bonding interactions viewed down different directions. Different colors are used for the two entangled frameworks. The $\mathrm{H}$ atoms involved in hydrogen bonding are emphasized in blue. The centroid-centroid distances for $\pi-\pi$ stacking are given in A. The parameters for hydrogen bonds are listed in Table S2.
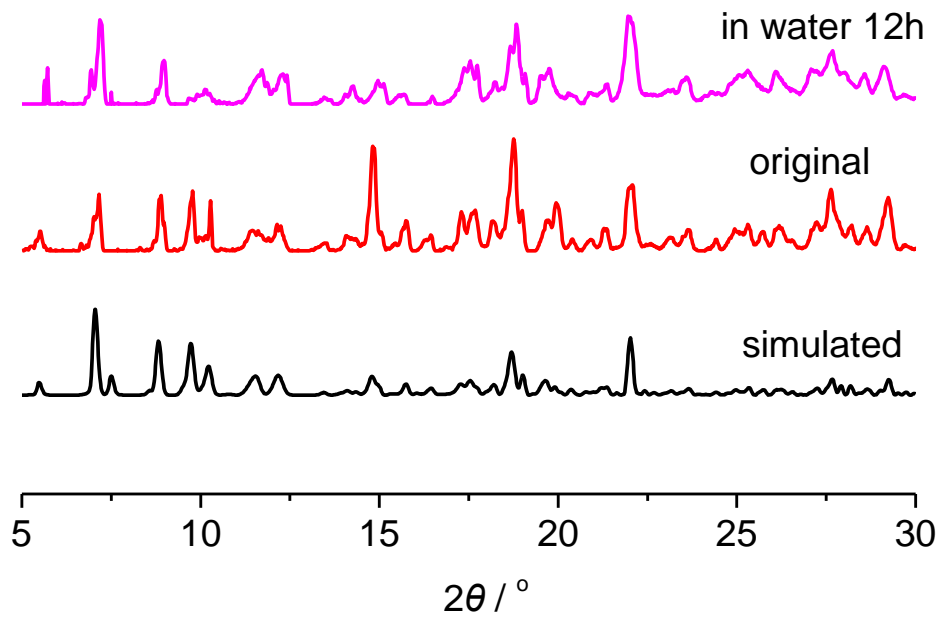

Figure S3. PXRD profiles of 1 after treated in water $12 \mathrm{~h}$. 


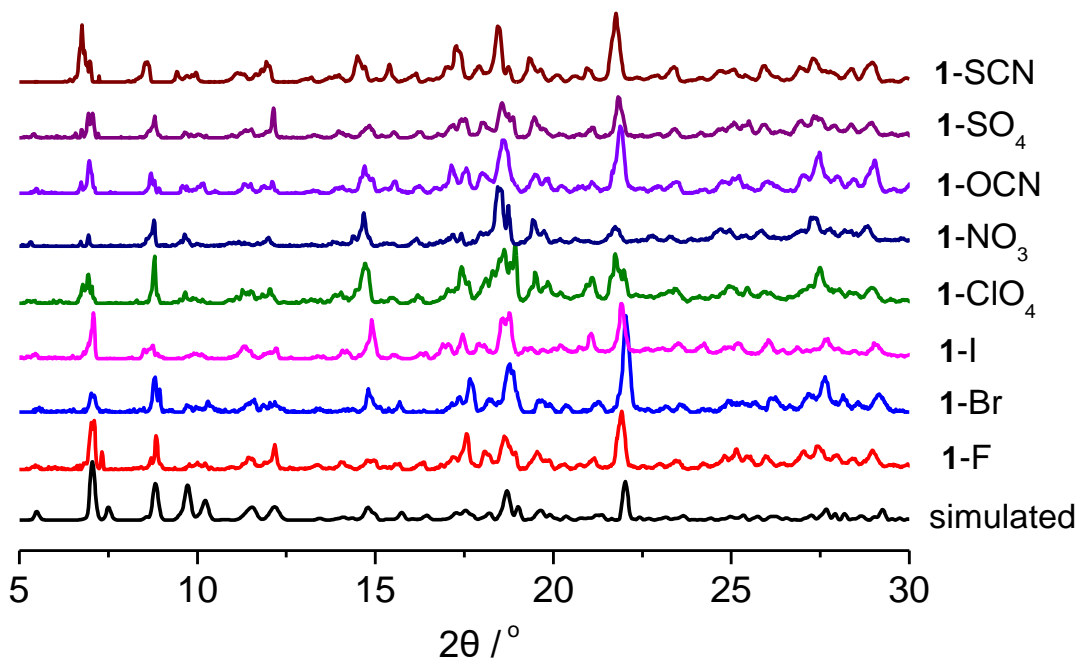

Figure S4. PXRD profiles of 1-X $\left(\mathrm{X}^{-}=\mathrm{SCN}^{-}, \mathrm{SO}_{4}{ }^{2-}, \mathrm{OCN}^{-}, \mathrm{NO}_{3}{ }^{-}, \mathrm{ClO}_{4}^{-}, \mathrm{I}^{-}, \mathrm{Br}^{-}\right.$, and $\left.\mathrm{F}^{-}\right)$.

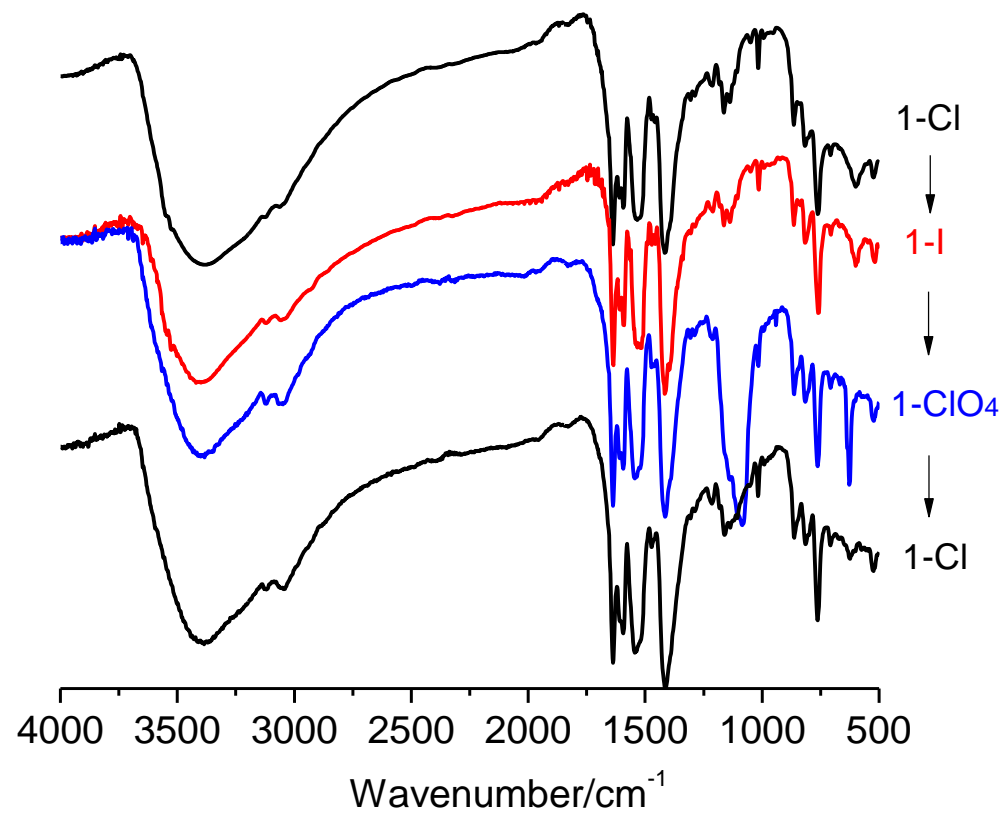

Figure S5. IR spectra of 1 after repeated exchange with $\mathrm{X}^{-}\left(\mathrm{X}^{-}=\mathrm{ClO}_{4}^{-}\right.$and $\left.\mathrm{I}^{-}\right)$. 


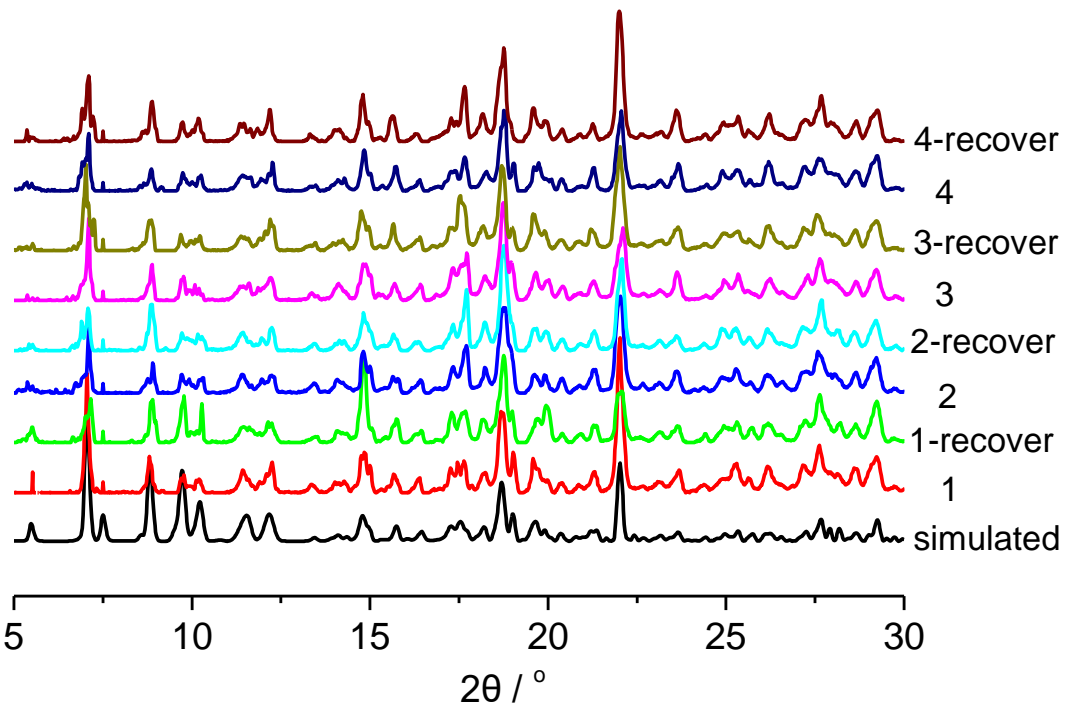

Figure S6. PXRD profiles of 1 durig four coloration and decoloration cycles with DEA.

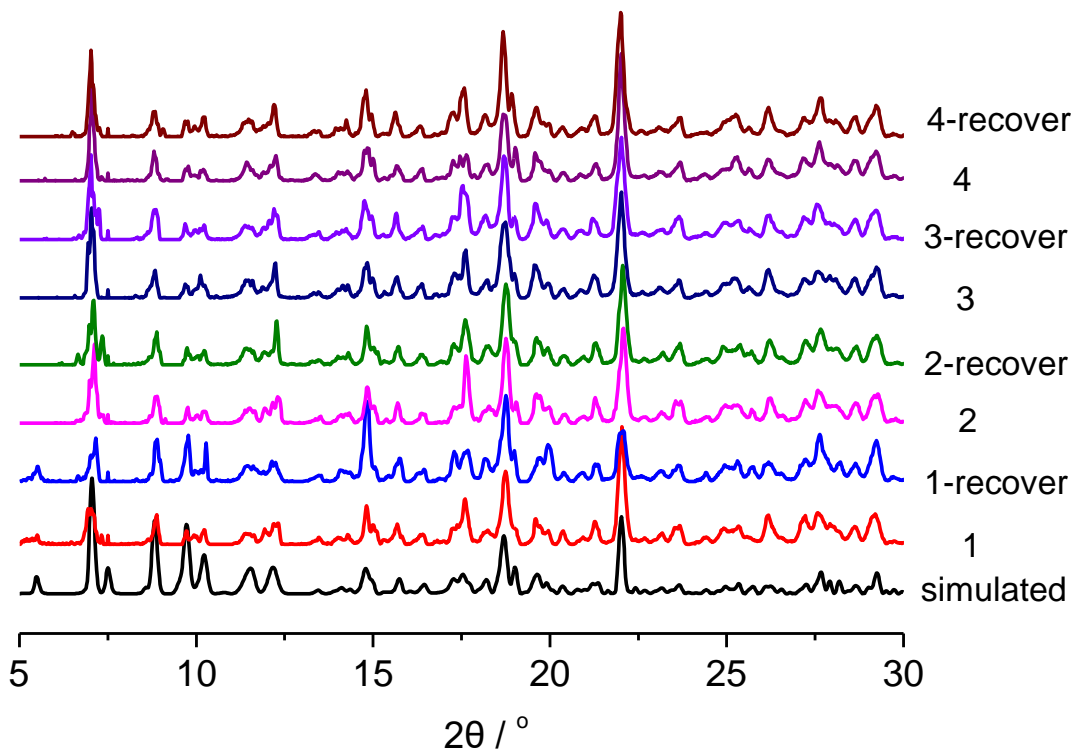

Figure S7. PXRD profiles of 1 during four coloration and decoloration cycles with EA.

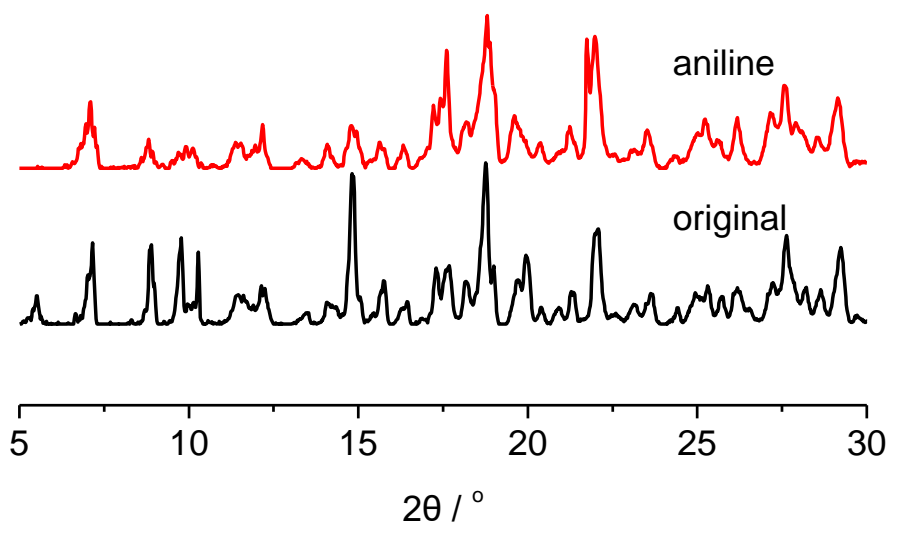

Figure S8. PXRD profiles of $\mathbf{1}$ after exposure to aniline. 
(a)

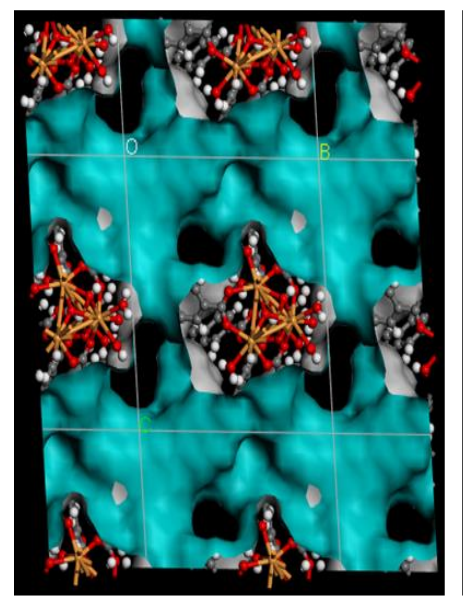

(b)

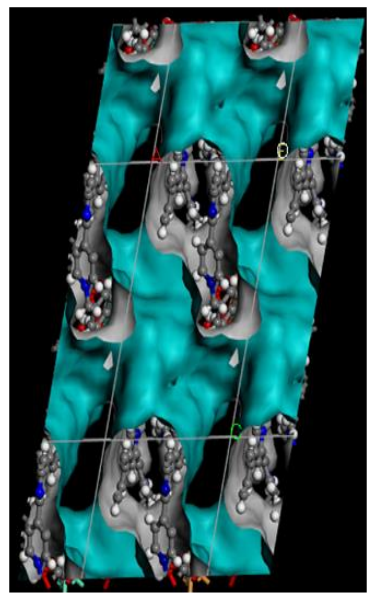

(c)

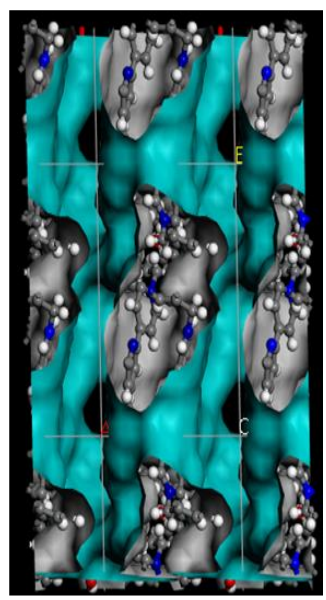

(d)

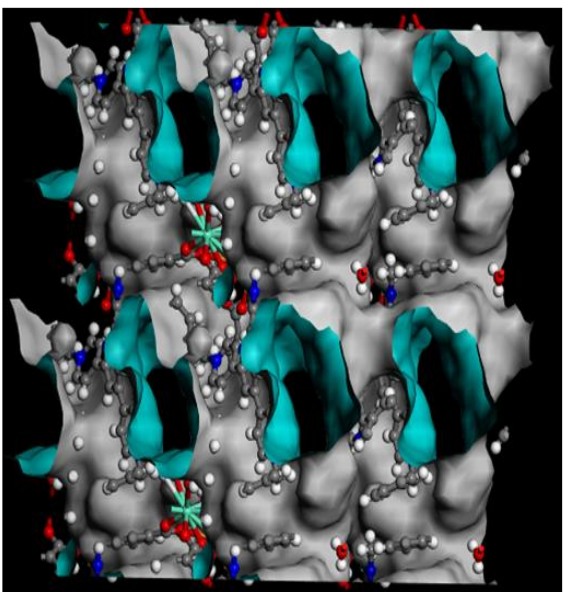

Figure S9. Connolly surfaces (Connolly radius $1.2 \AA$ ) showing the intercrossing pore system in 1. (a-c) Cross section profiles at $\mathrm{a}=0, \mathrm{~b}=0$ and $\mathrm{c}=-0.12$ down the $\mathrm{a}, \mathrm{b}$ and $\mathrm{c}$ axes, respectively. (d) The view of the channels down the (10-1) direction.

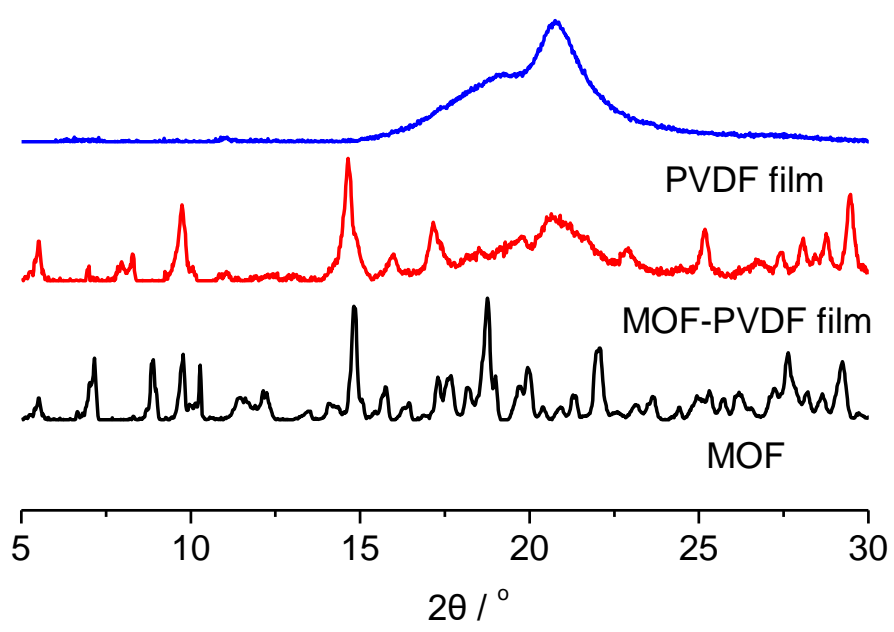

Figure S10. PXRD profile of the MOF-PVDF film compared with those of the MOF, and the PVDF film. 
(b) MOF-PVDF-film (50um)

(a) MOF-PVDF-film (photo)

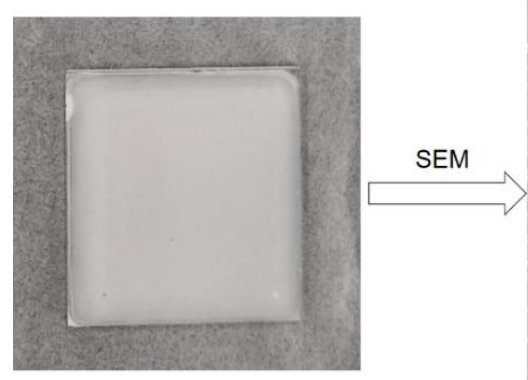

(c) EDX of MOF-PVDF-film

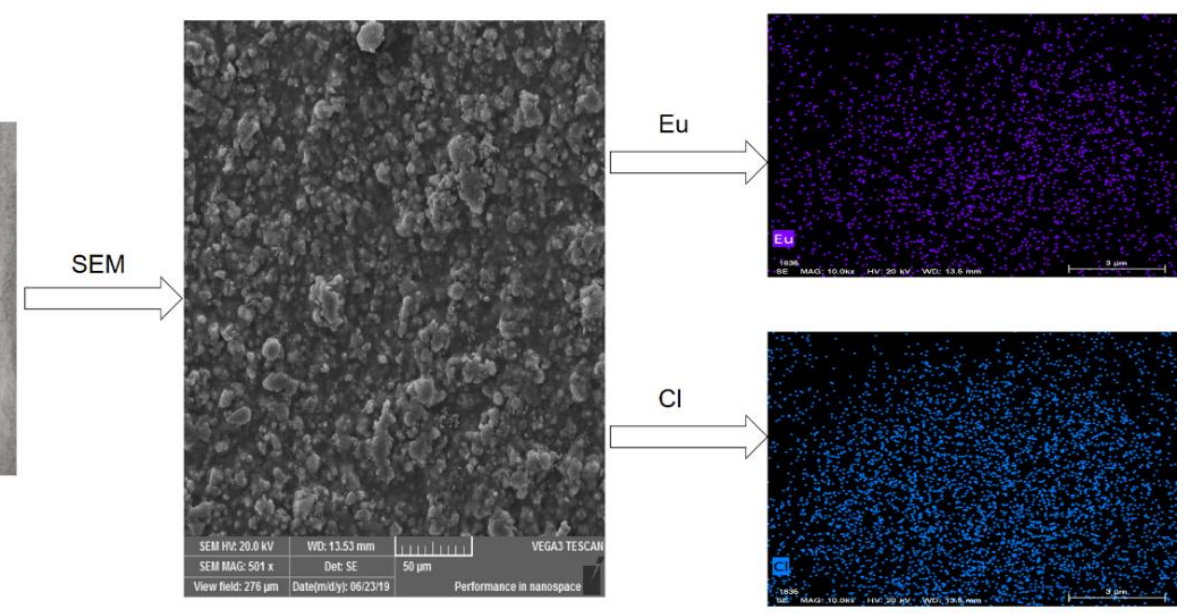

Figure S11. Photograph (a), SEM (b), and EDX (c) of the MOF-PVDF film.

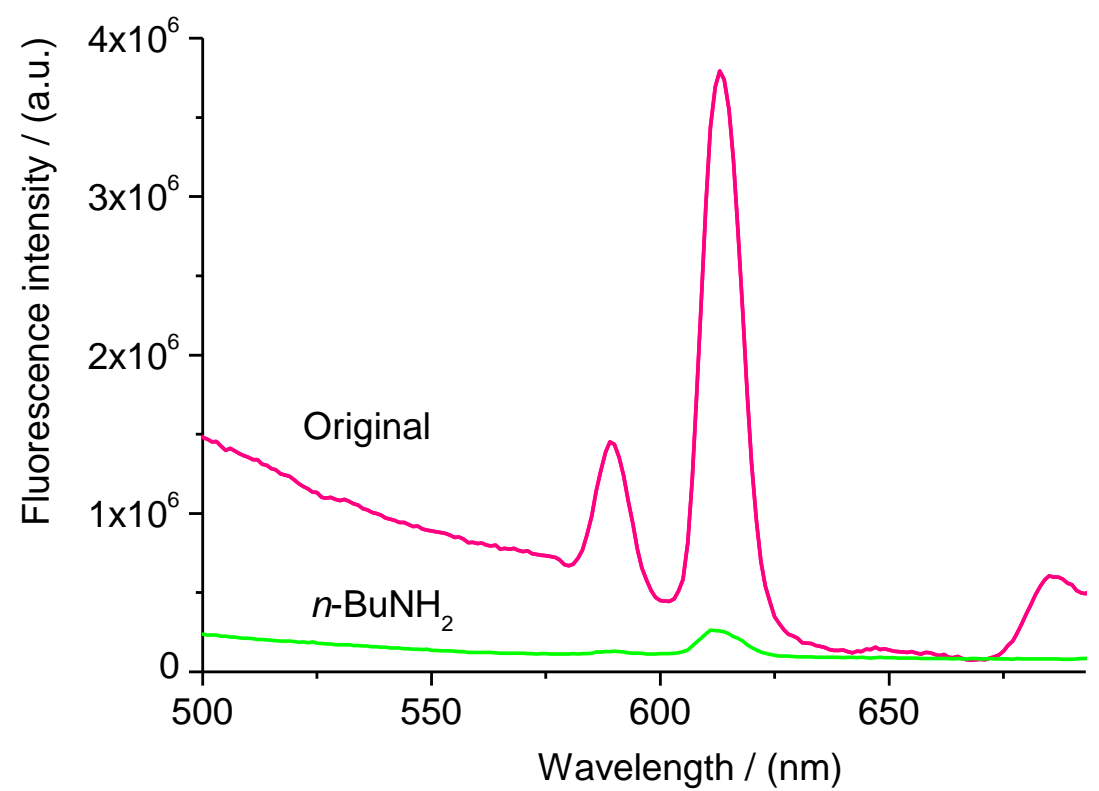

Figure S12. The emission spectra of the MOF-PVDF film before and after addition of $n$ $\mathrm{BuNH}_{2}\left(\lambda_{\mathrm{ex}}=358 \mathrm{~nm}\right)$.

\section{References:}

1. Sheldrick, G. M., SHELXTL. Bruker Analytical X-ray Instruments Inc.: Madison, Wisconsin, USA 1998.

2. Spek, A. L., PLATON SQUEEZE: a Tool for the Calculation of the Disordered Solvent Contribution to the Calculated Structure Factors. Acta Crystallogr., Sect. C: Struct. Chem. 2015, 71 (1), 9-18. 